Reprod. Nutr. Dévelop., 1985, 25 (3), 505-519.

\title{
Evolution postnatale de la composition en acides gras des lipides du tissu apideux et du tissu musculaire chez le lapin en régime hypolipidique.
}

\author{
J. OUHAYOUN, T. GIDENNE, Y. DEMARNE (*)
}

avec la collaboration technique de Viviane BATAILLER,

Véronique SANCHEZ, Muriel SEGURA, A. LAPANOUSE

Laboratoire de Recherches sur l'Elevage du Lapin

I.N.R.A., 31326 Castanet-Tolosan, Cedex, France.

(*) Station de Recherches de Nutrition, I.N.R.A., 78350 Jouy-en-Josas, France.

Summary. Postnatal changes in the fatty acid composition of adipose tissue and muscle tissue lipids of rabbits fed a low-fat diet.

The relative fatty acid profiles of perirenal adipose tissue and muscle tissue of the hind legs were studied in New Zealand rabbits 21 to 140 days old. The rabbits were fed a low-fat diet covering the essential fatty acid requirements and distributed either ad libitum or in rationed amounts.

The whole of the fatty acids were established earlier in hind leg muscle tissue than in perirenal adipose tissue. In the latter, the growth allometry of fatty acids in relation to the carcass was already higher between 21 and 74 days $(a=1.39$ ) and distinctly higher between 74 and 140 days $(a=2.68)$. In the muscle tissue, an isometric phase $(a=0.96)$ preceded a major allometric phase $(a=1.76)$.

In young suckling rabbits, the fatty acid profile of perirenal lipids was influenced by that of the milk lipids. In the muscle tissue, the presence of a large proportion of phospholipids resulted in a higher percentage of stearic acid and polyunsaturated fatty acids of the (n-6) family (linoleic, dihomo- $\gamma$-linolenic and arachidonic acids).

After weaning, the proportion of saturated fatty acids $(C \geq 14)$ in the perirenal adipose tissue remained constant. The endogenous production of monounsaturated fatty acids (higher allometry) caused a progressive dilution of polyunsaturated fatty acids (lower allometry). This dilution was slowed down when the diet was rationed.

Changes in the fatty acid composition of muscle tissue were, in general, comparable to those of perirenal adipose tissue. The differences observed could be attributed to the more active participation of phospholipids in the total lipids of the muscle tissue. Stearic acid, abundant in the phospholipids, showed a lower allometry compared to total fatty acids. Its dilution was an expression of triglyceride dilution of the phospholipids during growth. The same was true of polyunsaturated fatty acids. Rationing had the reverse effect.

\section{Introduction.}

La composition en acides gras des lipides corporels, en particulier celle des triglycérides, abondants dans le tissu adipeux, détermine les propriétés physicochimiques du tissu adipeux : aspect, profil de fusion, sensibilité aux processus 
d'oxydation. En production de viande, l'accumulation des acides gras au cours de l'élaboration des réserves lipidiques doit donc être maîtrisée. Chez les Monogastriques, la composition en acides gras des lipides corporels est fortement influencée par celle des matières grasses de la ration. L'effet des lipides alimentaires sur les lipides de réserve du Lapin, Monogastrique herbivore, a été décrit dans plusieurs expérimentations (Moore et Williams, 1968 ; Ciruzzi et al., 1973 ; Raimondi et al., 1975 ; Ouhayoun et al., 1981), mais aucune étude n'aborde, cians cette espèce, l'évolution de la composition en acides gras des lipides cor Norels au cours de la croissance lorsque l'aliment est exempt de lipides.

Le but de ce travail est de décrire l'installation de l'équilibre entre les acides gras après le sevrage chez des Lapins recevant un aliment n'apportant qu'un minimum d'acides gras essentiels, soit ad libitum pour favoriser la lipogenèse, soit de façon rationnée afin de limiter la mise en place des lipides de réserve.

\section{Matériel et méthodes.}

Matériel animal, conduite de l'expérimentation. - L'étude porte sur 152 lapins des deux sexes, de race Néozélandaise blanche, issus de 22 portées nées entre le 1 er et le 15 novembre 1982.

A l'âge de 21 jours, au moment où les lapereaux acquièrent la capacité de consommer de l'aliment sec, ils sont séparés de leur mère, sauf pendant le temps d'une têtée quotidienne. La mère continue à consommer l'aliment composé standard : les lapereaux ne disposent que de l'aliment expérimental hypolipidique. Le sevrage est complet à partir du $26^{\circ}$ jour postnatal. Les lapereaux sont maintenus par portée jusqu'à l'âge de 74 jours et alimentés à volonté. A l'âge de 74 jours, ils sont placés en cages individuelles; la moitié d'entre eux est rationnée. Ils ne reçoivent que les $2 / 3$ de la ration ingérée par les sujets alimentés à volonté. En moyenne quotidienne, la consommation d'aliment sec des lapins nourris à volonté est de $91,5 \mathrm{~g}$ entre 21 et 74 jours ; la vitesse de croissance est de $31,7 \mathrm{~g} / \mathrm{j}$. Pendant la deuxième phase de l'expérimentation (74-140 j), les lapins alimentés à volonté consomment en moyenne $158,6 \mathrm{~g} \pm 14,7 \mathrm{~g} / \mathrm{j}$; les lapins rationnés 109,2 $\pm 0,7 \mathrm{~g} / \mathrm{j}$. Les vitesses de croissance n'atteignent plus que $23,5 \mathrm{~g} / \mathrm{j}$ et $11,8 \mathrm{~g} / \mathrm{j}$ respectivement.

Echantillon expérimental. - Soixante cinq lapins sont sacrifiés à $21,30,39,52$, $74,90,106,122$ ou 140 jours, soit 5 lapins alimentés à volonté à chacun des 9 stades et 5 lapins rationnés aux quatre derniers stades. Ces lapins sont tirés au hasard parmi les individus dont le poids corporel est situé, au plus, à un écart type de la moyenne de l'ensemble de la population disponible lors de l'échantillonnage. Dans ces conditions, les caractères pondéraux des lapins sacrifiés ne diffèrent de ceux de la population d'origine à aucun des âges considérés.

L'influence du sexe n'a pas été prise en considération car, selon Vézinhet et Prud'hon (1975), le lapin mâle présente une grande similitude avec la femelle, en ce qui concerne l'adipogenèse totale et au niveau des diverses localisations, entre 10 et 182 jours d'âge. 
Aliments expérimentaux. - L'aliment consommé par les mères est standard. L'aliment expérimental, hypolipidique, est formulé de façon à présenter une teneur en lipides saponifiables plus basse encore que l'aliment standard $10,6 \mathrm{vs}$ $2,5 \%)$. L'huile de soja entrant dans la composition de ce mélange assure, avec l'huile contenue dans le tourteau de soja (1\% environ) un apport d'acide linoléique, acide gras essentiel, représentant, au maximum, $0,8 \%$ de l'énergie digestible $(3300 \mathrm{kcal} / \mathrm{kg})$. Les proportions des principaux acides gras des aliments sont données dans le tableau 1. Elles sont proches de celles de l'huile de soja, surtout en ce qui concerne l'aliment expérimental hypolipidique.

Caractères mesurés. - L'absence de carence en acides gras essentiels chez les lapins en croissance a été contrôlée par détermination du rapport de l'acide eicosatriénoïque $\left(C_{20}: 3, n-9\right)$ à l'acide arachidonique $\left(C_{20}: 4, n-6\right)$ dans les phospholipides hépatiques (Holman, 1968).

Les analyses de lipides concernent le tissu musculaire et le tissu adipeux qui diffèrent par leur précocité de croissance (Cantier et al., 1969). L'échantillonnage de tissu musculaire porte sur l'homogénat des muscles du membre postérieur débarrassés, le cas échéant, des filets adipeux intermusculaires. Cet ensemble représente environ $30 \%$ de la musculature chez le lapin âgé de 3 mois (Varewyck et Bouquet, 1982). Le tissu adipeux est prélevé au niveau périrénal.

Méthodes analytiques. - Les lipides sont extraits des tissus selon la technique de Folch, Lees et Sloane-Stanley (1957). Les acides gras sont séparés des autres constituants des lipides par saponification à froid, en présence d'un excès de potasse alcoolique. Les acides gras purifiés sont méthylés à chaud par le mélange méthanol, $\mathrm{HCl}(97: 3, \mathrm{~V} / \mathrm{V})$.

TABLEAU 1

Composition des aliments

\begin{tabular}{|c|c|c|}
\hline $\begin{array}{c}\text { Aliment standard } \\
\text { maternel }\end{array}$ & \multicolumn{2}{|l|}{$\begin{array}{c}\text { Aliment expérimental } \\
\text { hypolipidique }\end{array}$} \\
\hline Composition donnée par le fabri- & \multicolumn{2}{|l|}{ Composition centésimale } \\
\hline $\begin{array}{l}\text { cant: blé, son, luzerne, remoulage, } \\
\text { soja, tournesol, mélasse, CMV (Vit. } \\
\text { A, D, E, K, B1 et C) }\end{array}$ & $\begin{array}{l}\text { Paille de blé } \ldots \ldots \ldots \ldots \\
\text { Cellulose colmacel } \ldots \ldots \\
\text { Amidon purifié } \ldots \ldots \ldots \\
\text { Tourteau de soja } \ldots \ldots \ldots \\
\text { Huile de paraffine } \ldots \ldots \ldots \\
\text { Huile de soja } \ldots \ldots \ldots \ldots \\
\text { dl méthionine } \ldots \ldots \ldots \ldots \\
\text { CMV } 853 \text { (1) } \ldots \ldots \ldots \ldots\end{array}$ & $\begin{array}{r}20,0 \\
4,9 \\
36,0 \\
31,5 \\
3,0 \\
0,3 \\
0,3 \\
4,0\end{array}$ \\
\hline
\end{tabular}

Matières grasses $(\%)$

Proportions

des

acides gras

$1 \%$ des $A G$

totaux)
$C_{14}: 0$
$C_{16}: 0$
$C_{18}: 0$
$C_{18}:$
$C_{18}:$
$C_{18}: 3$
2,5

$3,6(2)$

$\begin{array}{rrr}C_{14}: 0 & - & 0,9 \\ C_{16}: 0 & 24,6 & 12,3 \\ C_{18}: 0 & 2,7 & 3,1 \\ C_{18}: 1 & 18,4 & 22,8 \\ C_{18}: 2 & 47,0 & 53,9 \\ C_{18}: 3 & 7,3 & 7,0\end{array}$

(1) Colin, Arkhurst et Lebas (1973) ; (2) dont 3,0\% d'huile de paraffine. 
L'analyse de la composition en acides gras des différents extraits est réalisée par chromatographie en phase gazeuse. Les conditions d'analyse sont les suivantes : colonne capillaire en verre de $0,3 \mathrm{~mm}$ de diamètre interne et de $47 \mathrm{~m}$ de longueur, appareil Girdel 75-FD-2, la phase stationnaire est du FFAP, le gaz vecteur est de l'hydrogène dont la pression au niveau de l'injecteur est de 0,5 bar, la température du four est de $196^{\circ} \mathrm{C}$, la détection est réalisée par ionisation de flamme. L'appareillage est couplé à un calculateur-intégrateur LTT 4212. Les différents acides gras séparés sont déterminés par calcul de leur longueur équivalente de chaîne (LEC).

De façon à mesurer l'importance des éventuelles sous-estimations des concentrations en acides gras à chaîne moyenne obtenues par cette méthode, un mélange de triglycérides dont la composition en acides gras est connue a subi toute la séquence des manipulations conduisant de l'extraction jusqu'à l'obtention des esters méthyliques. Dans nos conditions expérimentales, la sous-estimation n'est significative que pour les acides gras de longueur de chaîne inférieure à $C_{12}$.

Analyse mathématique des résultats expérimentaux. - L'évolution relative des caractères anatomiques et biochimiques au cours de la croissance est étudiée en utilisant la relation d'allométrie : $y=b x^{a}$. Le coefficient d'allométrie " a " représente le rapport des vitesses spécifiques de croissance des deux parties $y$ et $x$ de l'organisme :

$$
\frac{d y}{y d t} / \frac{d x}{x d t}
$$

ou la pente de la droite représentative de l'équation de régression de $\log _{\mathrm{e}} y$ sur $\log _{e} x: \log _{e} y=a \log _{e} x+\log b$.

La croissance relative du tissu musculaire du membre postérieur, du tissu adipeux périrénal et de leurs acides gras constitutifs totaux est étudiée en utilisant le poids de la carcasse, comme variable de référence $(x)$.

Les équations d'allométrie concernent :

- l'ensemble des lapins alimentés à volonté et sacrifiés entre 21 et 140 jours d'âge ;

- les lapins alimentés à volonté entre 21 et 74 jours ;

- chacun des groupes de lapins alimentés à volonté et rationnés entre 74 et 140 jours (sacrifices entre 90 et 140 jours).

Pour chacune des variables explicatives $(y)$ sont donnés :

- le coefficient d'allométrie (a) et son écart-type $\left(S_{a}\right)$,

- le coefficient de corrélation de l'ajustement linéaire ( $r$ ) et sa signification statistique (F),

- les valeurs extrêmes de la variable expliquée $(x)$ entrant dans l'ajustement (données naturelles),

- la valeur de $\log b$.

Les coefficients d'allométrie homologues sont comparés selon la méthode de Dagnélie (1970).

L'évolution de la composition en acides gras du tissu musculaire et du tissu adipeux est étudiée en prenant comme variable de référence les acides gras totaux de ces deux tissus, respectivement. 


\section{Résultats.}

Croissance relative des composants de la carcasse (tabl. 2 et 3 ).

Coefficients d'allométrie moyens entre 21 et 140 jours d'âge chez les lapins alimentés à volonté. - Par rapport à la carcasse, prise comme variable de référence, l'allométrie des muscles du membre postérieur est faiblement majorante.

TABLEAU 2

Allométrie de croissance des constituants de la carcasse (poids en g)

\begin{tabular}{|c|c|c|c|c|c|c|c|}
\hline & & & $\begin{array}{c}\text { Tissu } \\
\text { musculaire } \\
\text { membre } \\
\text { postérieur }\end{array}$ & $\begin{array}{c}\text { Tissu } \\
\text { adipeux } \\
\text { périrénal }\end{array}$ & $\begin{array}{l}\text { AG totaux } \\
\text { t. musculaire } \\
\text { membre } \\
\text { postérieur }\end{array}$ & $\begin{array}{l}\text { AG totaux } \\
\text { tissu } \\
\text { adipeux }\end{array}$ & $\begin{array}{c}\text { Bornes de } \\
X\end{array}$ \\
\hline \multirow{3}{*}{$\begin{array}{l}\text { Lapins } \\
\text { alimentés } \\
\text { à volonté }\end{array}$} & $\begin{array}{c}21 \text { à } \\
140 \\
\text { jours }\end{array}$ & $\begin{array}{c}a \pm S_{a} \\
\stackrel{r}{\log b}\end{array}$ & $\begin{array}{c}1,11 \\
\pm 0,01 \\
0,998 \\
-3,066\end{array}$ & $\begin{array}{r}1,72 \\
\pm 0,08 \\
0,961 \\
-8,918\end{array}$ & $\begin{array}{r}0,95 \\
\pm 0,05 \\
0,939 \\
-5,793 \\
\end{array}$ & $\begin{array}{r}1,72 \\
\pm 0,08 \\
0,955 \\
-9,247 \\
\end{array}$ & $197-2237$ \\
\hline & $\begin{array}{c}21 \text { à } \\
74 \\
\text { jours }\end{array}$ & $\begin{array}{c}a \pm S_{a} \\
r \\
\log b\end{array}$ & $\begin{array}{c}1,14 \\
\pm 0,01 \\
0,998 \\
-3,243\end{array}$ & $\begin{array}{r}1,33 \\
\pm 0,14 \\
0,899 \\
-6,642\end{array}$ & $\begin{array}{c}0,96 \\
\pm 0,11 \\
0,881 \\
-5,807\end{array}$ & $\begin{array}{c}1,39 \\
\pm 0,16 \\
0,880 \\
-7,282\end{array}$ & $197-1355$ \\
\hline & $\begin{array}{c}90 \text { à } \\
140 \\
\text { jours }\end{array}$ & $\begin{array}{c}a \pm S_{a} \\
r \\
\log b\end{array}$ & $\begin{array}{r}1,25 \\
+0,09 \\
0,960 \\
-4,078\end{array}$ & $\begin{array}{r}2,36 \\
\pm 0,32 \\
0,869 \\
-13,583\end{array}$ & $\begin{array}{r}1,76 \\
+0,25 \\
0,857 \\
-11,882\end{array}$ & $\begin{array}{r}2,68 \\
+0,35 \\
0,874 \\
-16,384\end{array}$ & $1083-2237$ \\
\hline $\begin{array}{l}\text { Lapins } \\
\text { rationnés }\end{array}$ & $\begin{array}{c}90 \text { à } \\
140 \\
\text { jours }\end{array}$ & $\begin{array}{c}a \pm S_{a} \\
r \\
\log b\end{array}$ & $\begin{array}{r}1,18 \\
\pm 0,07 \\
0,965 \\
-3,471 \\
\end{array}$ & $\begin{array}{r}2,60 \\
\pm 0,94 \\
0,547 \\
-15,913\end{array}$ & $\begin{array}{c}0,77 \\
\pm 0,42 \\
0,393^{\text {NS }} \\
-4,537\end{array}$ & $\begin{array}{r}2,60 \\
\pm 1,00 \\
0,520 \\
-16,245\end{array}$ & 1 126-1 734 \\
\hline
\end{tabular}

NS : non significatif $(P>0,1) ;{ }^{* *}: 0,01<P<0,05 ; * * *: P<0,01$.

Les coefficients de corrélation ne portant pas la mention NS sont hautement significatifs $(P<0,01)$.

TABLEAU 3

Evolution de la composition des carcasses entre 21 et 140 jours (valeurs moyennes en g)

\begin{tabular}{|c|c|c|c|c|c|c|}
\hline \multirow{2}{*}{ Régime } & \multirow{2}{*}{ Age } & \multirow{2}{*}{$\begin{array}{l}\text { Poids } \\
\text { carcasse }\end{array}$} & \multicolumn{2}{|c|}{ Muscles du membre postérieur } & \multicolumn{2}{|c|}{ Tissu adipeux périrénal } \\
\hline & & & Poids & Acides gras & Poids & Acides gras \\
\hline \multirow{4}{*}{ Ad libitum } & 21 & 218 & 18,2 & 0,44 & 2,4 & 1,67 \\
\hline & 74 & 1155 & 123,5 & 2,45 & $\begin{array}{r}17,9 \\
17,9\end{array}$ & 1394 \\
\hline & $\begin{array}{l}14 \\
90\end{array}$ & 1580 & 168,6 & 2,68 & 36,9 & 24,69 \\
\hline & 140 & 2110 & 236,2 & 5,65 & 97,7 & 74,56 \\
\hline \multirow{2}{*}{ Rationné } & 90 & 1280 & 145,5 & 2,75 & 16,5 & 11,61 \\
\hline & 140 & 1661 & 192,6 & 3,45 & 46,3 & 34,28 \\
\hline
\end{tabular}


L'évolution relative des acides gras totaux du tissu musculaire est pratiquement isométrique de celle de la carcasse. Le tissu adipeux périrénal et ses acides gras présentent le même coefficient d'allométrie élevé.

Discontinuité de la croissance relative. - Entre les deux périodes de la croissance considérées (21-74 jours, d'une part, 90-140 jours, d'autre part), le coefficient d'allométrie du tissu musculaire ne varie pas significativement $(a>1)$. En revanche, les coefficients d'allométrie du tissu adipeux périrénal et de ses acides gras sont plus élevés au cours de la seconde période de la croissance. Cela est vrai aussi, mais moins nettement, pour ce qui concerne les acides gras du tissu musculaire.

Influence du rationnement entre 74 et 140 jours. - Le ralentissement de la vitesse de croissance pondérale globale obtenu par rationnement ne modifie pas significativement l'allométrie de croissance du tissu musculaire. Celle du tissu adipeux périrénal et de ses acides gras constitutifs reste majorante, mais les écartstypes des coefficients d'allométrie sont considérablement augmentés. L'ajustement linéaire n'est plus vérifié en ce qui concerne les acides gras musculaires.

\section{Evolution relative des acides gras du tissu adipeux périrénal.}

L'acide laurique $\left(\mathrm{C}_{12}: 0\right)$ disparaît des lipides dès l'âge de 39 jours. Les acides myristique $\left(C_{14}: 0\right)$, palmitique $\left(C_{16}: 0\right)$ et stéarique $\left(C_{18}: 0\right)$ évoluent à la même vitesse relative que l'ensemble des acides gras périrénaux. Cette isométrie est vérifiée, tant sur l'ensemble de la période étudiée ( 21 à 140 jours) qu'au cours de chacune des deux phases successives (21 à 74 jours, 90 à 140 jours). Le rationnement ne modifie en rien le phénomène (tabl. 4). Il en résulte une bonne constance du taux des acides gras saturés. Dès l'âge de 21 jours, leur équilibre définitif est atteint (fig. 1).

Chez les lapins alimentés à volonté, les acides gras monoénoïques : acides palmitoléique $\left(C_{16}: 1, n-7\right)$ et oléique $\left(C_{18}: 1, n-9\right)$, présentent une allométrie majorante. En revanche, les acides gras diénoïques : acides linoléique $\left(C_{18}: 2, n-6\right)$ et linolénique $\left(\mathrm{C}_{18}: 3, n-3\right)$, se distinguent par une allométrie nettement minorante. Ces caractéristiques des acides gras insaturés sont constantes tout au long de la période étudiée. Par conséquent, entre 21 et 140 jours, les taux des acides gras monoinsaturés, surtout de l'acide palmitoléique, augmentent régulièrement, alors que les taux des acides gras polyinsaturés décroissent. Le rationnement appliqué à une partie des lapins à partir de 74 jours n'a pas d'effet significatif sur les coefficients d'allométrie. Toutefois, il tend à ralentir l'accumulation relative des acides gras monoinsaturés et à augmenter celle des acides gras polyinsaturés. Mais cela ne se traduit pas par une évolution sensible des taux des acides gras mono et polyinsaturés.

\section{Evolution relative des acides gras du tissu musculaire.}

Entre 21 et 140 jours, les acides gras du tissu musculaire se mettent en place à des vitesses relatives très différentes chez les lapins alimentés à volonté.

L'acide laurique subsiste jusqu'à l'âge de 90 jours, puis disparaît. Les acides myristique et palmitique présentent la même évolution relative que l'ensemble des acides gras, avec une tendance à l'allométrie majorante. Mais l'acide stéarique se distingue des autres acides gras saturés par une allométrie minorante (tabl. 5). 


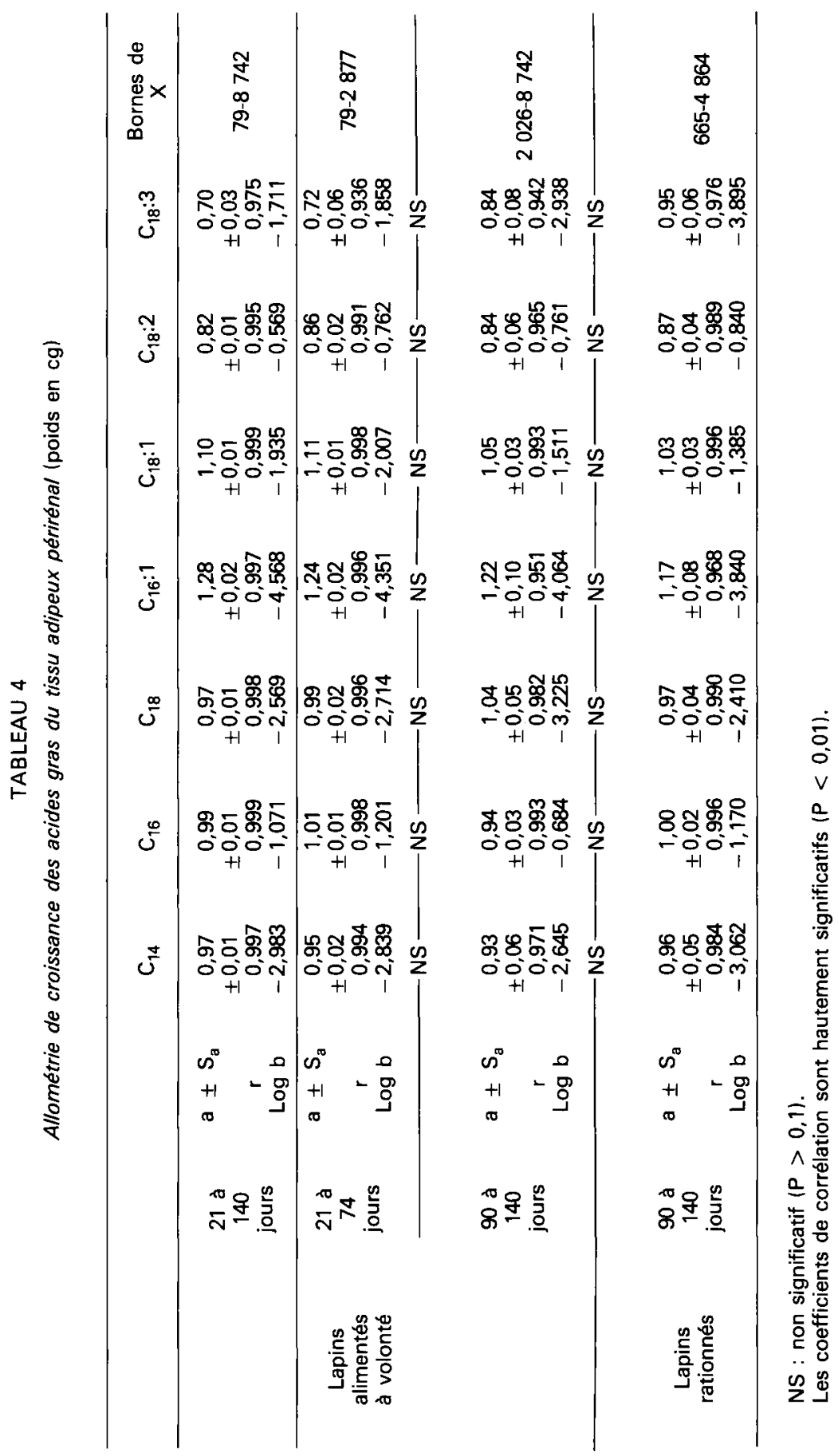




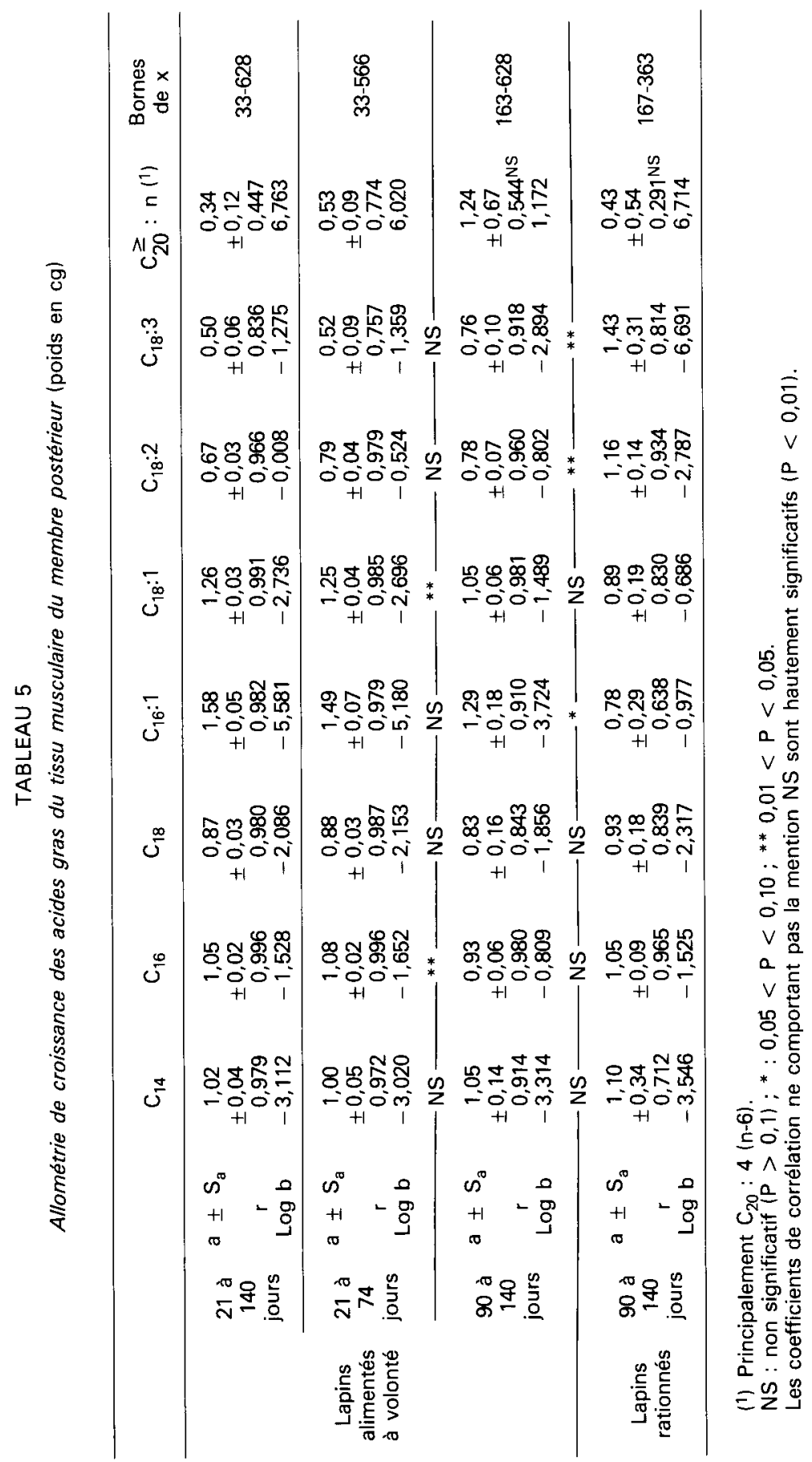




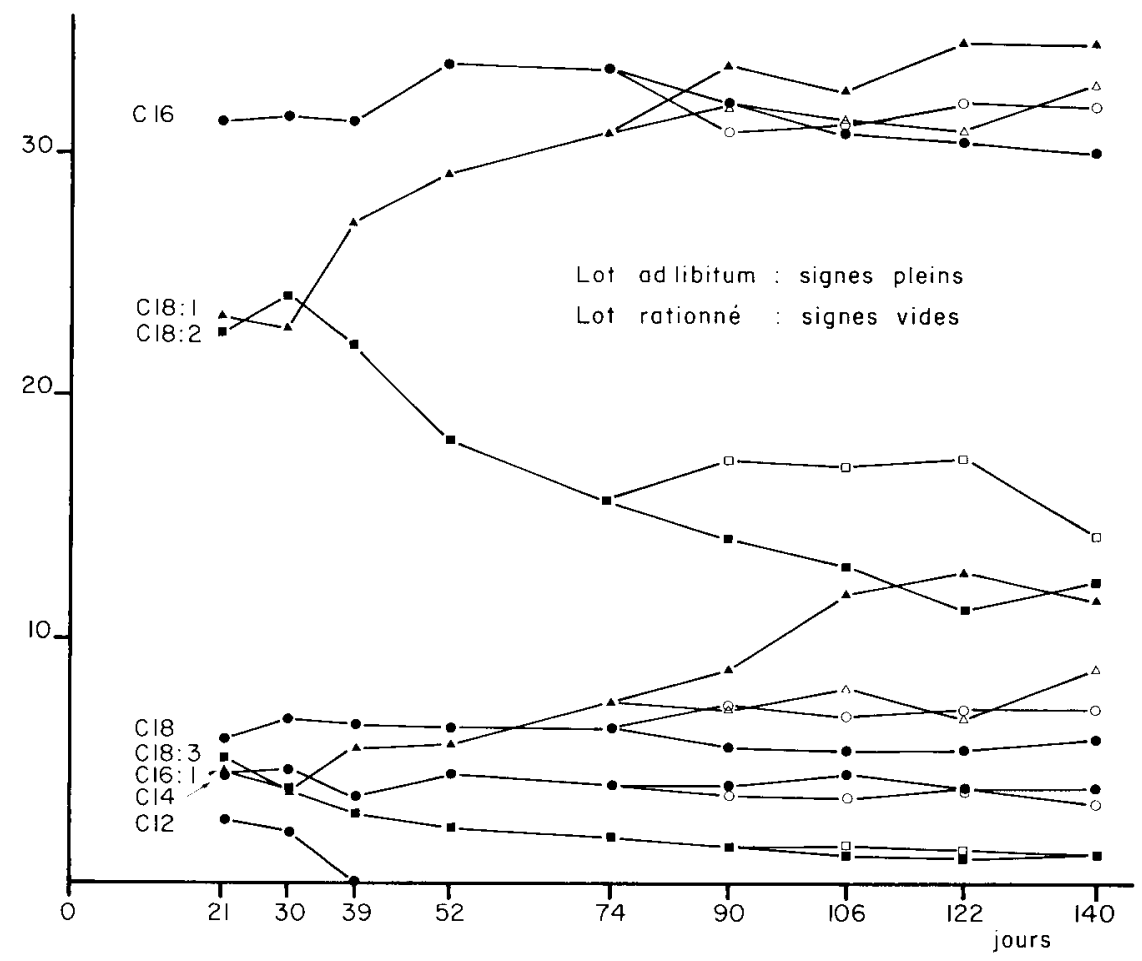

FIG. 1. - Evolution de la composition en acides gras du tissu adipeux périrénal (\% des esters méthyliques dosés).

L'acide palmitoléique et, à un moindre degré, l'acide oléique, présentent une allométrie majorante. L'allométrie de croissance des acides gras polyinsaturés, qu'il s'agisse de l'acide linoléique, de l'acide linolénique et surtout de l'ensemble des acides arachidonique $\left(C_{20}: 4, n-6\right)$ et dihomo- $\gamma$ linolénique $\left(C_{20}: 3, n-6\right)$ est nettement minorante.

Par conséquent, entre le sevrage et l'âge de 140 jours, les proportions des acides gras sont profondément modifiées (fig. 2). L'évolution observée est attribuable surtout aux acides gras monoinsaturés lacide palmitoléique et acide oléique), dont le pourcentage global double. En revanche, les acides gras polyinsaturés sont fortement déprimés; le taux des acides gras de la famille (n-6) : acide linoléique, acide dihomo- $\gamma$-linolénique, acide arachidonique passe de 25 à $13 \%$. L'acide $\alpha$ linolénique $\left(C_{18}: 3, n-3\right)$ est aussi très dilué $(1,2 \%$ vs $4,3 \%)$.

Ces évolutions ne sont pas continues. En effet, l'acide palmitique présente une allométrie majorante entre 21 et 74 jours puis minorante par la suite. Une évolution semblable concerne l'acide oléique : une première phase d'allométrie fortement majorante est suivie d'une phase d'isométrie. Mais aucun changement d'allométrie n'affecte l'acide linoléique, principal acide gras polyinsaturé. Au-delà de l'âge de 74 jours, la teneur des lipides musculaires en acides gras polyinsaturés comptant au moins 20 atomes de carbone est très faible ; la liaison linéaire entre 
ce groupe d'acides gras et l'ensemble des acides gras musculaires n'est alors plus vérifiée.

Le rationnement entre 74 et 140 jours n'intervient pas significativement sur les coefficients d'allométrie des acides gras saturés : une légère hausse est toutefois généralement observée. L'allométrie de croissance des acides gras monoinsaturés, de l'acide palmitoléique surtout, devient minorante. En revanche, l'allométrie de croissance des acides gras polyinsaturés, minorante chez les lapins nourris à volonté, devient nettement majorante chez les lapins rationnés.

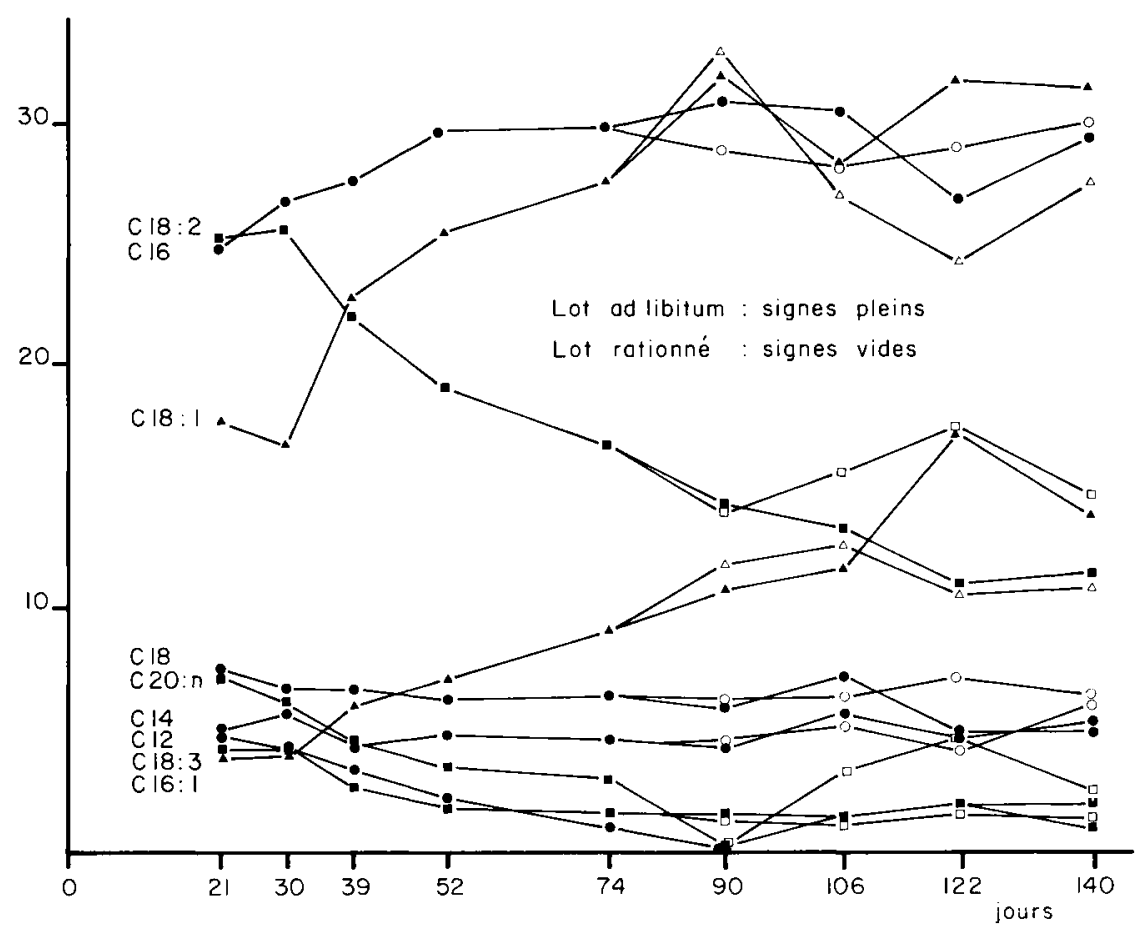

FIG. 2. - Evolution de la composition en acides gras du tissu musculaire (\% des esters méthyliques dosés).

\section{Discussion.}

Eléments de composition corporelle.

L'ordre de précocité décroissante des tissus musculaires puis adipeux, qui est déduit du classement par ordre croissant des coefficients d'allométrie moyens de ces tissus, dans l'intervalle 21-140 jours, chez les lapins alimentés à volonté est classique.

Le changement d'allométrie du tissu adipeux périrénal (phases d'allométrie majorante puis fortement majorante) est conforme aux résultats de Cantier et al. (1969), portant sur l'ensemble du tissu adipeux dissécable de lapins d'une souche commune. 
Alors que, selon ces mêmes auteurs, la croissance relative de la musculature répond à une phase d'allométrie majorante, puis à une phase d'allométrie minorante, les muscles des membres postérieurs considérés ici présentent une allométrie constamment majorante. Cela résulte probablement de la précocité relativement faible de ces groupes musculaires due à leur situation à la convergence de deux gradients de croissance musculaire, l'un antéro-postérieur, l'autre distoproximal, remontant de l'extrémité de la jambe vers la ceinture pelvienne (Cantier et al., 1974).

L'évolution parallèle des coefficients d'allométrie du tissu adipeux périrénal et de ses acides gras au cours des deux périodes (21-74 jours et 90-140 jours) est expliquée par le fait que ces derniers représentent, selon les stades, de 67 à $78 \%$ de la masse du tissu adipeux. La méthode d'étude utilisée ne permet pas de mettre en évidence la contribution prépondérante successive des phénomènes d'hypertrophie et d'hyperplasie des adipocytes à l'adipogenèse au cours de la croissance (Nouguès, 1975 ; Nouguès et Vézinhet, 1977).

Les acides gras musculaires sont impliqués, à la fois, dans des phospholipides, abondants dans la viande de lapin particulièrement pauvre en matières grasses (Kim et Kim, 1982) et dans les triglycérides. Entre 21 et 74 jours, la valeur du coefficient d'allométrie des acides gras du tissu musculaire $(a=0,96)$ plus faible que celle du tissu musculaire $(a=1,14)$ résulte, d'une part, de la dilution des phospholipides de structure au cours de la croissance des fibres musculaires dont le nombre est définitivement fixé 30 jours après la naissance (Nouguès, 1972), d'autre part, de la décroissance, dans le tissu musculaire, des mécanismes oxydatifs utilisant les lipides comme substrat à des fins énergétiques, ce qui correspond à une dilution des mitochondries (Bacou, 1972; Ouhayoun, Delmas et Poujardieu, 1982). Entre 90 et 140 jours, l'allométrie nettement majorante des acides gras musculaires est une expression du développement de cellules adipeuses intramusculaires et de l'établissement de réserves de triglycérides. En fait, ces derniers n'ont pas une localisation uniquement intramusculaire car les fines inclusions adipeuses intermusculaires ne sont pas totalement éliminées par dissection. L'écart existant entre les coefficients d'allométrie moyens des acides gras du tissu musculaire $(a=0,95)$ et du tissu adipeux périrénal $(a=1,72)$ calculés sur une période longue (21 à 140 jours) suggère une mise en place plus précoce des acides gras du tissu musculaire.

Cette hypothèse est étayée par le fait que les acides gras musculaires se révèlent moins sensibles à l'influence du rationnement alimentaire que les acides gras périrénaux. On sait, en effet, que le rationnement énergétique affecte préférentiellement les éléments corporels formés tardivement. Ainsi, dès l'âge de 90 jours, c'est-à-dire 16 jours après le début du rationnement, le poids moyen des carcasses des lapins rationnés ( $R$ ) est inférieur à celui des lapins alimentés ad libitum (A).

L'écart relatif $\frac{R-A}{A}$ entre les moyennes s'élève à - $19 \%$. Les acides gras musculaires (écart relatif : $+2,6 \%$ ) et le tissu musculaire $(-13,7 \%$ ) sont peu modifiés alors que le tissu adipeux périrénal $(-55,3 \%)$ et ses acides gras 
(- $53 \%$ ) sont nettement déprimés. A l'âge de 140 jours, l'effet différentiel du rationnement sur ces éléments est confirmé.

\section{Equilibre des acides gras.}

Dans la mesure où l'aliment expérimental est pratiquement lipidoprive - les lipides saponifiables représentent moins de $0,8 \%$ de l'énergie digestible de la ration - l'évolution de la composition en acides gras des tissus au cours de la croissance dépend essentiellement de la dilution par les acides gras d'origine endogène des acides gras présents à l'issue de la période d'allaitement. Le processus de dilution, caractéristique de chaque acide gras, dépend de son implication dans les molécules de lipides (triglycérides ou phospholipides) et de leur vitesse de renouvellement.

\section{Influence du lait.}

Pendant les 3 premières semaines postnatales, les lapereaux ne consomment que du lait : celui-ci est très riche en lipides ( $39 \%$ de la matière sèche); chacun d'eux reçoit, en moyenne, $3 \mathrm{~g}$ /jour de matières grasses (Lebas, 1971).

A priori, la composition en acides gras du lait de lapine est très différente de celle des lipides des tissus du lapereau âgé de 21 jours. En effet, les acides gras comptant 10 atomes de carbone ou moins représentent les $2 / 3$ des acides gras du lait (Demarne et al., 1978) ; ils sont absents des lipides du jeune. En fait, celui-ci utilise les acides gras courts à des fins énergétiques. Une grande similitude apparaît entre le lait et les lipides du jeune sous la mère lorsque seuls les acides gras ayant au moins 12 atomes de carbone sont pris en considération. Toutefois, l'acide laurique est plus abondant dans le lait que dans les lipides corporels (15\% vs 3 à $5 \%$ ); seul le tissu musculaire contient des acides gras à 20 ou 22 atomes de carbone en quantités significatives. II s'agit principalement de l'acide arachidonique $\left(C_{20}: 4, n-6\right)$.

Pendant la quatrième semaine postnatale, alors que la production laitière de la lapine décroît, la teneur en lipides du lait augmente : chaque lapereau consomme alors $4 \mathrm{~g}$ de lipides par jour (Lebas, 1971). Dans le même temps, l'apport de matières grasses provenant de l'aliment sec, qui est consommé à partir du $21^{\mathrm{e}}$ jour, ne représente pas $5 \%$ du total.

Au sevrage (26 jours) correspond un changement qualitatif dans l'apport d'acides gras alimentaires mais surtout un changement quantitatif. La formation de triglycérides à partir d'acides gras d'origine essentiellement endogène entraîne la formation de nouveaux équilibres.

Dans le tissu adipeux périrénal, le taux des acides gras saturés $(C \geq 14)$ reste inchangé. L'acide myristique, absent de l'aliment expérimental, se caractérise par un taux proche de celui qui est observé dans le suif des Ruminants ; il est largement supérieur à celui des autres Monogastriques (Adrian, Legrand et Frangne, 1981). La stabilité de la proportion de l'acide palmitique et de son produit d'élongation, l'acide stéarique, contraste avec la progression régulière du taux des deux acides gras monoinsaturés majeurs : en particulier de l'acide palmitoléique, qui provient de la désaturation de l'acide palmitique, et de l'acide oléique, issu de 
l'acide stéarique. L'acide palmitoléique est d'origine strictement endogène, ce qui n'est pas le cas de l'acide oléique ( $23 \%$ environ des acides gras de l'aliment). Cependant, la synthèse endogène de ce dernier excède largement l'apport alimentaire. En effet, en considérant que le tissu adipeux périrénal, entre 21 et 140 jours, représente, en moyenne, le quart du tissu adipeux total (d'après Vézinhet et Prud'hon, 1975), et en faisant l'hypothèse, vérifiée chez le Rat (Gandemer, 1979) que l'acide oléique présente le même taux dans les diverses localisations adipeuses, le tissu adipeux dissécable, à lui seul, accumule 5 fois plus d'acide oléique que n'en apporte l'aliment dans la même période. Chez les lapins rationnés, l'accumulation d'acide oléique dans le tissu adipeux est encore 3 fois plus élevée que son ingestion.

Malgré la faible teneur en matières grasses de l'aliment, les lapins alimentés à volonté ingèrent une quantité non négligeable d'acide linoléique : $0,38 \mathrm{~g} / \mathrm{jour}$, en moyenne, entre 21 et 140 jours, mais nettement plus faible d'acide linolénique : $0,05 \mathrm{~g} / \mathrm{j}$. Ces apports ne sont pas suffisants pour assurer le maintien de leurs taux initiaux ( 21 jours). Chez les lapins rationnés, les apports d'acides gras exogènes sont réduits (environ $69 \%$ de l'ingestion à volonté) mais, étant donné que la production d'acides gras monoinsaturés endogènes est moins active, la dilution des acides gras polyinsaturés est plus lente.

L'équilibre initial des acides gras est sensiblement différent dans le tissu musculaire. Les acides gras polyinsaturés sont plus abondants, aux dépens des acides gras mono-insaturés. Le plus faible taux de ces derniers est aussi attribuable au niveau élevé de l'acide stéarique. Le profil des acides gras à l'âge de 21 jours est fortement dépendant des phospholipides de structure. L'évolution post-sevrage de ce profil résulte, à la fois, des mécanismes observés dans le tissu adipeux, où sont impliqués presque exclusivement les triglycérides, et de la dilution des phospholipides par les triglycérides. Ce second facteur explique la baisse du taux d'acide stéarique et la diminution particulièrement rapide des acides linoléique et linolénique, au cours de la croissance des lapins alimentés à volonté.

En freinant la formation de réserves de triglycérides dans le tissu musculaire, ce qui accroît la part des phospholipides dans les lipides totaux, le rationnement ralentit la diminution du taux d'acide stéarique et améliore la représentation des acides gras polyinsaturés. Les acides palmitoléique et oléique jouent un rôle tampon dans ces évolutions.

Chez des lapins en croissance âgés de 11 semaines, ayant reçu depuis leur sevrage un aliment hypolipidique, l'équilibre des acides gras constitutifs des lipides du tissu adipeux périrénal et du tissu musculaire se révèle donc très différent de celui qui est observé chez des lapins de même âge engraissés avec des aliments du commerce. Ces aliments sont généralement pauvres en matières grasses ( 2 à $4 \%$ ), mais celles-ci se caractérisent par une teneur élevée en acides linolénique (galactolipides des chloroplastes de la luzerne) et linoléique (tourteau de soja). Près du tiers des acides gras du tissu adipeux périrénal et du tissu musculaire est alors polyinsaturé (Ouhayoun et al., 1981) ; l'acide linoléique représente environ $20 \%$ de l'ensemble des acides gras; dans le tissu adipeux périrénal, l'acide linolénique atteint le taux de $7 \%$ des acides gras; l'acide arachidonique 
produit de la désaturation et de l'élongation de l'acide linoléique est présent à raison de $4 \%$ des acides gras du tissu musculaire.

Enfin, chez des lapins en régime hypolipidique, l'influence du profil en acides gras des lipides du lait est marquée et durable même lorsque la distribution à volonté de l'aliment est favorable à la lipogenèse endogène. Bien entendu, l'aliment expérimental semi-synthétique n'a pas d'intérêt en production, mais il fournit une base utile aux études en cours portant sur les possibilités de substitution partielle de lipides aux glucides alimentaires chez le lapin de boucherie.

Reçu en septembre 1984. Accepté en janvier 1985.

Remerciements. - Etude réalisée avec l'aide financière de la DGRST (contrat 81 C 1242).

\section{Références}

ADRIAN J., LEGRAND G., FRANGNE R., 1981. Dictionnaire de biochimie alimentaire et de nutrition, 222-225. Technique et Documentation, Paris.

BACOU F., 1972. Evolution quantitative de l'aldolase, de l'aspartate aminotransférase, de la succinate déshydrogénase et de l'acétylcholinestérase dans les muscles blancs et rouges du Lapin au cours de la période postnatale. C.R. Soc. Biol. Montpellier, 1037-1042.

CANTIER J., VÉZINHET A., ROUVIER R., DAUZIER L., 1969. Allométrie de croissance chez le Lapin. I/Principaux organes et tissus. Ann. Biol. anim. Bioch. Biophys., 9, 5-39.

CANTIER J., VÉZINHET A., DULOR J. P., ROUVIER R., 1974. Allométrie de croissance chez le Lapin. IV/Principaux muscles de la carcasse. Ann. Biol. anim. Bioch. Biophys., 14, 271-292.

CIRUZZI B., MINOIA P., BUFANO G., MUSCIO A., 1973. Caratteristiche chimiche delle carni e del grasso in conigli di diverse razza. Ann. Fac. agrar., Univ. Bari, 26, 797-815.

COLIN M., ARKHURST G., LEBAS F., 1973. Effets de l'addition de méthionine au régime alimentaire sur les performances de croissance chez le Lapin. Ann. Zootech., 22, 485-491.

DAGNÉLIE P., 1970. Théorie et méthodes statistiques. Vol. 2, 281-287. Ed. Duculot, Gembloux.

DEMARNE Y., LHUILLERY C., PIHET J., MARTINET L., FLANZY J., 1978. Comparative study of triacylglycerol fatty acids in milk fat from two leporidae species : rabbit (Oryctolagus cuniculus) and hare (Lepus europeus). Comp. Biochem. Physiol., 61, 223-226.

FOLCH J., LEES M., SLOANE-STANLEY G. H., 1957. A simple method for the isolation and purification of total lipids from animal tissues. J. Biol. Chem., 226, 497-509.

GANDEMER G., 1979. Contribution à l'étude de la lipogenèse de novo chez le Rat. Th. Doct. Ing., I.N.A. Paris-Grignon, $80 \mathrm{pp}+\mathrm{X}$.

HOLMAN R. T., 1968. The ratio of trienoic: tetraenoic acids in tissue lipids as a measure of essential fatty acid requirement. J. Nutr., 70, 405-410.

KIM C. H., KIM Y. H., 1982. Studies on lipids and fatty acids composition of various meats. Kor. J. anim. Sci., 24, 452-456.

LEBAS F., 1971. Composition chimique du lait de lapine. Evolution au cours de la traite et en fonction du stade de lactation. Ann. Zootech., 20, 185-191.

MOORE J. H., WILLIAMS D. L., 1968. The effect of diet on the composition and positional distribution of the fatty acids in the triglycerides obtained from the adipose tissues of rabbits. Brit. J. Nutr. 22, 473-482.

NOUGUĖS J., 1972. Etude de l'évolution du nombre des fibres musculaires au cours de la croissance postnatale du muscle chez le Lapin. C.R. Soc. Biol., 166, 165.

NOUGUĖS J., 1975. Adipocyte growth of four adipose deposits in rabbit (NZ). Ann. Biol. anim. Bioch. Biophys., 15, 541-546.

NOUGUES J., VEZZINHET A., 1977. Evolution pendant la croissance de la cellularité du tissu adipeux chez le Lapin et l'Agneau. Ann. Biol. anim. Bioch. Biophys., 17, 765-952. 
OUHAYOUN J., DELMAS D., POUJARDIEU B., 1982. Variabilität der Myoglobinkonzentration im Kaninchenmuskel-Beziehungen zum Energiestoffwechsel. II. Int. Koll. "Das Kaninchen als Modeltier und Züchtungsobjekt ", Sept., Rostock.

OUHAYOUN J., DEMARNE Y., DELMAS D., LEBAS F., 1981. Utilisation de pellicules de colza dans l'alimentation du Lapin en croissance. II/Effet sur la qualité des carcasses. Ann. Zootech., 30, 325-333.

RAIMONDI R., MARIA C. de, AUXILIA M. T., MASOERO G., 1975. Effect of adding fat to the feed on meat production in rabbits. 3/Fatty acid composition of meat and perirenal fat. Ann. Istit. sper. Zootec., 8, 167-181.

VAREWYCK H., BOUQUET Y., 1982. Relations entre la composition tissulaire de la carcasse de lapins de boucherie et celle des principaux morceaux. Ann. Zootech., 31, 257-268.

VÉZINHET A., PRUD'HON M., 1975. Evolution of various adipose deposits in growing rabbits and sheeps. Anim. Prod., 20, 363-370. 